\title{
Axialization of Laser Cooled Magnesium Ions in a Penning Trap
}

\author{
H. F. Powell, D. M. Segal, and R. C. Thompson \\ Blackett Laboratory, Imperial College, Prince Consort Road, London SW7 2BW, United Kingdom
}

(Received 8 March 2002; published 12 August 2002)

\begin{abstract}
We report the first demonstration of the axialization of laser cooled ions in a Penning trap. Axialization involves the application of a small radial quadrupole drive which couples the cyclotron and magnetron motions. This enhances the laser cooling, allowing tighter confinement of the ions to the central axis of the trap than is otherwise possible. Using an intensified charge-coupled device (ICCD) camera we have imaged the axialization process for the first time. For a single ion, we recorded a large decrease of the magnetron amplitude corresponding to a reduction in ion temperature of approximately 2 orders of magnitude to an upper limit of order $10 \mathrm{mK}$. We have discovered dynamics specific to the laser cooled system which depend critically on the axial drive frequency and amplitude.
\end{abstract}

DOI: 10.1103/PhysRevLett.89.093003

With the growing interest in quantum information processing there has been an increasing need to develop technology which might enable the implementation of the ideas proposed. The uniquely stable and isolated environment of the ion trap makes it an ideal tool for the investigation of quantum phenomena; indeed, ion traps have been placed at the forefront of advances in the area of quantum information science [1]. Recent experiments include the entanglement of four trapped ions, the building of a CNOT gate and quantum state engineering using a single particle [2-4].

Very low temperatures can be reached in ion traps using laser cooling, but the minimum temperature can be limited by the nature of the trapping method itself. The two most widely used types of ion trap are the Paul (radio frequency) trap and the Penning trap [5]. For radial confinement, the former uses an electric field oscillating at a radio frequency, while the latter uses a static magnetic field. This means that in the Paul trap the ion motion is simple harmonic in all directions but in the radial plane of the Penning trap the action of the combined electric and magnetic fields results in a more complex orbital motion: a superposition of two rotations, the magnetron and the cyclotron rotations.

Until now much work in the area of quantum information science has focused on the use of linear Paul traps which allow the preparation of stationary strings of ions and strong confinement to the Lamb-Dicke regime [1]. Unfortunately, the low temperature limit of the Paul trap can be affected by the "micromotion" of the trapped ions, which arises as a result of the trapping field and cannot be completely avoided. The Penning trap has no such limitation [5]. However, the nature of the motion in the radial plane of the Penning trap complicates the laser cooling process [6,7]. Specifically, it is difficult to achieve small magnetron radii. Problems arise because the magnetron motion is unstable: its total energy is negative so energy must be supplied in order to reduce the magnetron radius.

Three frequencies govern the motion of ions in a Penning trap. The first is $\omega_{z}$, which describes the simple
PACS numbers: 32.80.Pj, 03.67.Lx, 41.20.-q, 42.50.Vk

harmonic motion in the axial direction. The other two are associated with the radial motion: the modified cyclotron frequency, $\omega_{c}^{\prime}$, and the magnetron frequency, $\omega_{m}$. These frequencies depend upon the trapping voltage, $V_{t}$, with the sum of the magnetron and modified cyclotron frequencies always being equal to the true cyclotron frequency, $\omega_{c}=$ $e B / m$ (where $e$ is the electronic charge, $m$ the ion mass, and $B$ the magnetic field strength). In our trap, the frequencies have been measured for ${ }^{24} \mathrm{Mg}^{+}\left(V_{t}=10 \mathrm{~V}\right.$, $B=0.97 \mathrm{~T})$ to be $\omega_{z}=283 \mathrm{kHz}, \omega_{m}=72 \mathrm{kHz}$, and $\omega_{c}^{\prime}=557 \mathrm{kHz}$.

Axialization is the process by which particles in a Penning trap are driven towards the central axis of the trap [8]. It occurs when the efficient cooling of the cyclotron motion is effectively extended to the magnetron motion by coupling between the two induced by a weak external field oscillating at the true cyclotron frequency, $\omega_{c}$. This makes the conditions within the Penning trap approach those of the Paul trap, but without micromotion. As a result, it is expected that very low temperatures may be reached. This makes the Penning trap a serious contender for quantum information processing applications, especially where a magnetic field is required [9].

The greatest interest in axialization has been in the field of Fourier transform-ion cyclotron resonance (FT-ICR) spectroscopy. In these experiments the trapped ions are buffer gas cooled and development of the cyclotron driving technique has produced improved experimental methods [10]. The mass selectivity and longer storage times enabled [11] have led to the possibility for increased precision measurement of fundamental constants, and atomic $g_{J}$ values [12].

Here we are concerned with a laser cooled system of a few ions. The process of laser cooling in a Penning trap is a well analyzed problem $[6,7,13]$. Instability of the magnetron motion means that it is difficult to decrease both the magnetron and the cyclotron amplitudes simultaneously. It can be shown that the magnetron motion is reduced only when the laser is placed at the point where the rotation moves the ions in the same direction as the laser photons. 
Even so the cyclotron motion is minimized considerably more efficiently than the magnetron orbit. This is due to the relatively small gradient of laser intensity across the center of the trap [7].

The motional frequencies of trapped ions can be probed by measuring the response of the ion cloud to a small oscillating drive voltage applied to the trap electrodes. In a laser cooled system the changing shape of the ion cloud as a result of resonant driving is reflected in the fluorescence intensity emitted by the ions. By scanning a range of drive frequencies close to one of the ion motional frequencies a dip or peak will be observed in the fluorescence at the resonant frequency. Drive voltages can be applied to the trap electrodes in a number of configurations, which offer different ways of manipulating and detecting the cloud oscillations.

Of interest here is a scheme termed azimuthal quadrupolar excitation: axialization. This requires that the ring electrode be split radially into four electrically isolated segments each of which is supplied with an oscillating voltage of frequency, $\omega_{c}$. A phase difference of $\pi$ is introduced between the supplies to adjacent segments. The quadrupole field allows energy to be coupled between the modified cyclotron and magnetron motions [8]. With the additional presence of suitable damping, the radius of the magnetron motion decays, leaving the ions axialized. In this state the ion cloud becomes insensitive to the laser beam position which can now be moved to the trap center for the most efficient ion confinement.

An alternative technique for increasing the density of ion cloud plasmas in a Penning trap is the "rotating wall," where the cloud rotation frequency $\left(\omega_{r}=\omega_{c} / 2\right.$ for maximum plasma density) is locked to an external drive [14]. Sympathetic cooling can also be used to reach low temperatures and compress an ion cloud [15]. Our method is particularly well suited to small clouds and especially single ions for use in quantum information processing.

The equations of motion for our system are $[16,17]$

$$
\dot{r}_{c}=\delta r_{m}-\gamma_{c} r_{c}, \quad \dot{r}_{m}=-\delta r_{c}-\gamma_{m} r_{m},
$$

where $r_{c}$ and $r_{m}$ are the radii of the cyclotron and the magnetron orbits, respectively, $\gamma_{c}$ and $\gamma_{m}$ are the corresponding damping coefficients, and $\delta$ is the coupling constant of the axializing drive. Assuming that $r_{c}$ and $r_{m}$ evolve as $\exp (-\Gamma t)$, we obtain an expression for the damping coefficient $\Gamma$ :

$$
\Gamma=\left[\left(\gamma_{c}+\gamma_{m}\right) \pm \sqrt{\left(\gamma_{c}+\gamma_{m}\right)^{2}-4\left(\gamma_{c} \gamma_{m}+\delta^{2}\right)}\right] / 2 .
$$

Consideration of this expression leads to the following four distinguishable conditions: (i) If $\gamma_{c}=\gamma_{m}=0$ the system oscillates between pure cyclotron and pure magnetron motion with no reduction in energy. (ii) If either $\gamma_{c}$ or $\gamma_{m}$ is large and positive (with the other one small) such that $\delta^{2}>-\gamma_{c} \gamma_{m}$, then $r_{c}, r_{m} \rightarrow 0$ as $t \rightarrow \infty$. (iii) If $\delta^{2}=$ $-\gamma_{c} \gamma_{m}$ with $\gamma_{c}>\delta>0, \gamma_{m}<0$, then we obtain a stable orbit such that $r_{m} / r_{c}=\gamma_{c} / \delta=\left|\delta / \gamma_{m}\right|$. (iv) If $\delta^{2}<$ $-\gamma_{c} \gamma_{m}$, then the orbit expands until condition (iii) is met. Axialization occurs only when condition (ii) is met. The final set of conditions embodies the main difference between buffer gas cooled and laser cooled systems. With buffer gas cooling, the damping coefficients have a fixed ratio and are constant for a given buffer gas pressure. With laser cooling, the ratio of the two damping rates (and even their signs) can be varied by changing the laser frequency and/or position. In addition, both damping rates reduce if the motion of the ions takes them outside the laser beam. Therefore the stable orbit mentioned above can be achieved only in the case of laser cooling [17].

The experiments were carried out using magnesium ions which can be laser cooled using the ${ }^{2} S_{1 / 2} \rightarrow{ }^{2} P_{3 / 2}$ transition at $280 \mathrm{~nm}$. The light is provided by a stabilized, frequency doubled dye laser pumped by an $\mathrm{Ar}^{+}$laser working on a single line at $514 \mathrm{~nm}$. The trap is described in Ref. [18]. Laser light passes through the trap via holes cut into the ring electrode, and light from the ions emerges from a hole also cut in the ring. The fluorescence signal is focused into a photomultiplier tube and photon counts are recorded using a multichannel scaler. In some investigations the counts from the photomultiplier were sent to a time to amplitude converter so that photon-photon correlations could be analyzed [19]. To image using the intensified charge-coupled device (ICCD) camera, some light is picked off and focused onto the camera array with a magnification of $\sim 1$. In this experiment the exposure time for each image is $10 \mathrm{~s}$.

To produce an axialized cloud the trap is initially loaded with a small number of ions. With the $280 \mathrm{~nm}$ laser frequency detuned by approximately $400 \mathrm{MHz}$ the cyclotron drive is applied at a peak to peak amplitude of $0.4 \mathrm{~V}$. If the signal is monitored by the photomultiplier, an increase in fluorescence is observed. This increase in the count rate is immediate and can be explained by the cloud becoming more dense when the drive is applied: its Doppler width and radial extent being reduced by the drive field. Maximum signal and full axialization are achieved when the laser frequency approaches resonance and the beam is central within the trap.

When the drive frequency is scanned from below to above the cyclotron frequency, a resonance peak can be observed in the ion fluorescence. It was found that the width of the cyclotron resonance depends upon the position of the laser beam. Figure 1 shows the cyclotron resonance for a single ion (confirmed by monitoring quantum jumps). Initially (trace 1) when the laser beam is in its usual position for laser cooling, the resonance is broad: $20 \mathrm{kHz}$ (FWHM). Once the beam position is moved to the center of the trap (trace 2) the resonance narrows: $<200 \mathrm{~Hz}$ (FWHM). Reduction of the resonance width occurs because, with the laser beam centered, the magnetron motion 

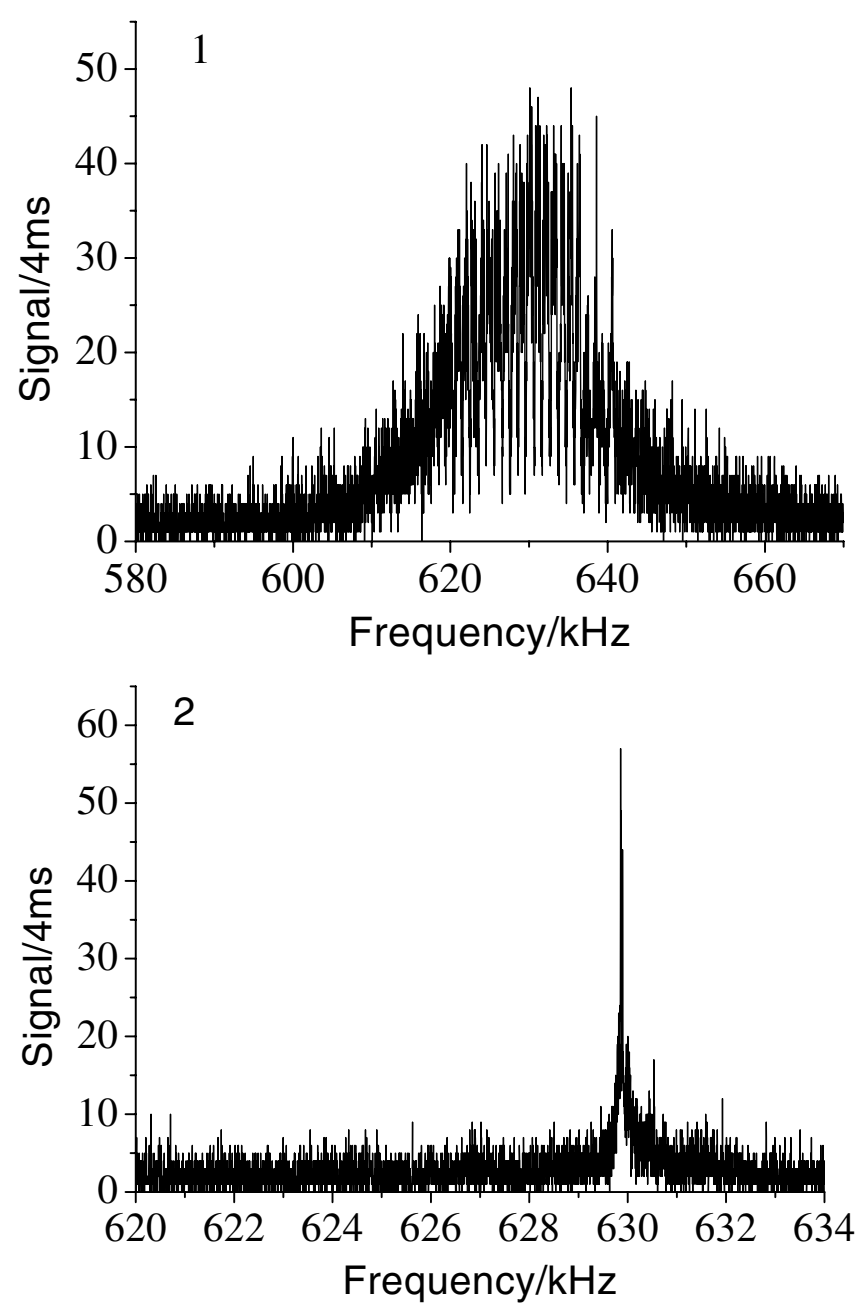

FIG. 1. Alteration in cyclotron resonance width with the laser beam position for a single ion (see text). Drive voltage amplitude, $V_{d}$, was optimized to obtain the narrowest resonance without significantly weakening the response. For trace $1, V_{d}=$ $30 \mathrm{mV}$, and for trace $2, V_{d}=141 \mathrm{mV}$. (The sharp downward spikes in trace 1 are an artifact of the signal generator used.)

is cooled only when the drive is present at the cyclotron frequency.

Using the ICCD camera, the process of axialization can be observed. Referring to Fig. 2: image 1 shows a laser cooled cloud with no drive present, while image 2 shows the same cloud immediately after the drive is applied. There is an instant reduction in cloud radius by a factor of 2.4. Optimizing the laser parameters further decreases the cloud radius.

The volume occupied by a cold ion cloud is determined by its plasma rotation frequency and the ion number [20]. In the limit of a single ion, however, the plasma description is no longer applicable. In this case, we can speak of reduction of the magnetron radius as "cooling," and the concept of an effective temperature can be associated with the size of the magnetron orbit.

The modified cyclotron motion is directly cooled by the laser; thus the radius of this orbit is always smaller than
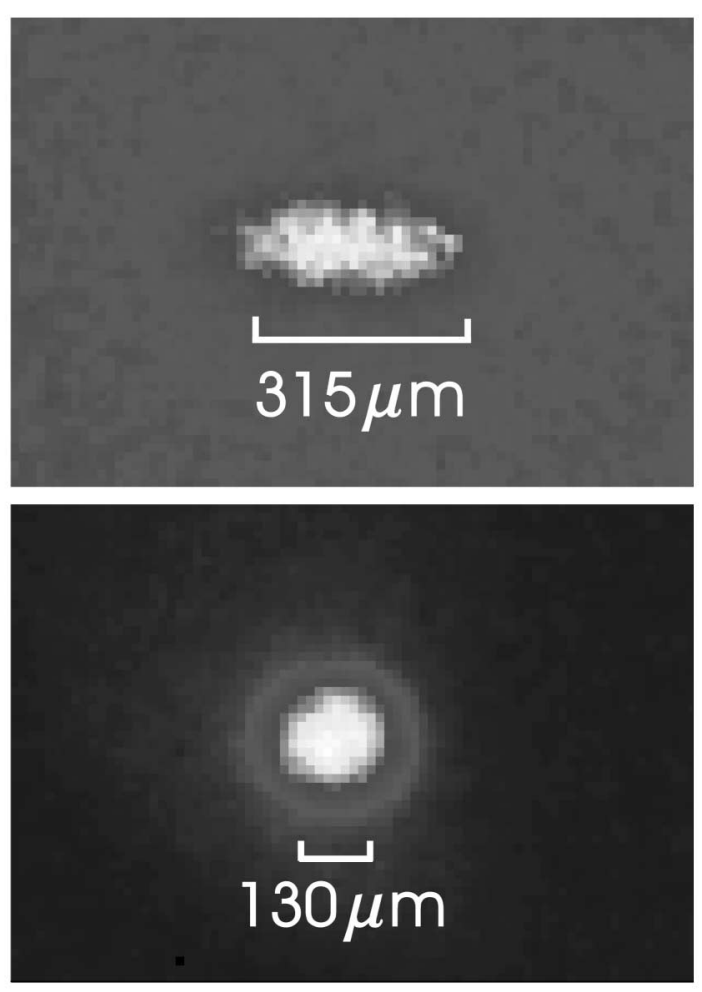

2

FIG. 2. Application of the cyclotron drive to a small ion cloud. From the fluorescence rate, the cloud size is $\sim 5$ ions. Laser detuning and beam position are the same for both images. The dimensions given correspond to the image plane.

that of the magnetron. We can then assume that the extent of an ion orbit in the radial plane is principally due to the radius of the magnetron motion, so the measured size of the radial orbit can be used to calculate an upper limit for the temperature of the ion.

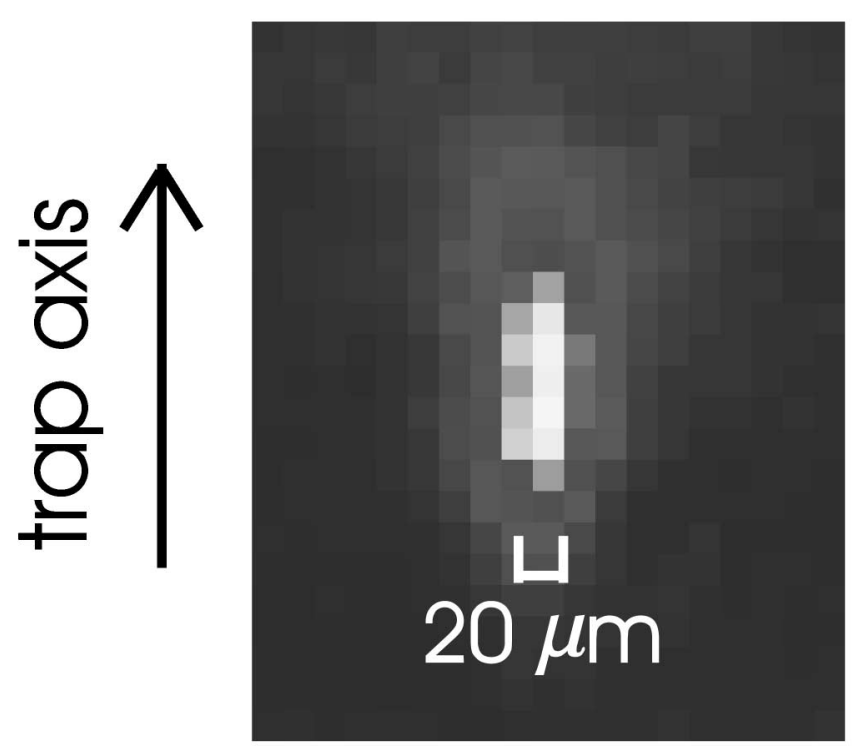

FIG. 3. A single axialized ion. Here the radial motion is of smaller amplitude than the axial. 


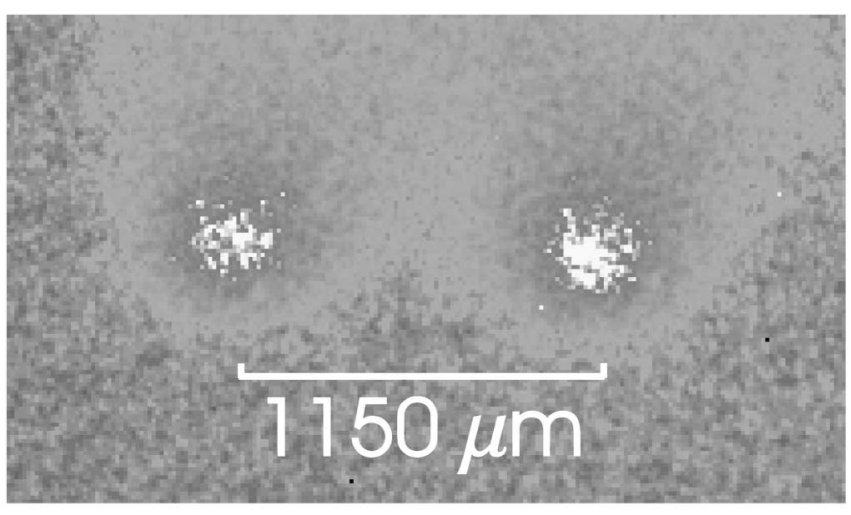

FIG. 4. A cloud of approximately 50 ions in a large stable orbit.

In the absence of an axializing drive the radial extent of a single ion trajectory indicates a temperature of order $\sim 1 \mathrm{~K}$. A single, axialized, ion is shown in Fig. 3. In the radial direction the size of the image is $20 \mu \mathrm{m}$ (1.5 pixels). Allowing for the finite resolution of the camera, this gives an estimate for the ion temperature of order $10 \mathrm{mK}$. In our trap geometry the axial motion receives no direct cooling; thus its amplitude and therefore its temperature is greater than in the radial plane. Aberrations in the imaging optics will tend to increase the image size; thus all temperature calculations should be considered an upper limit.

Larger clouds have been trapped and axialized. Images of these clouds show the minimum radius scaling upwards with increasing ion numbers. This is in accordance with the limitations on maximum cloud density imposed by space charge effects.

Some interesting phenomena have been noted. In particular, we were able to observe stable orbits of ions when the laser cooling was strong compared to the coupling rate [as predicted by conditions (iii) and (iv) above]. These orbits are seen as two fluorescence spots, showing where the circular path of the ions interacts with the laser beam, as shown in Fig. 4. Photon-photon correlation spectra show that the ions are in a single cold cloud, orbiting the trap at the magnetron frequency, rather than a ring of ions as might be expected. Further details of these investigations will be given in a separate publication [17].

In conclusion, we have demonstrated and imaged the axialization of laser cooled ions in a Penning trap for the first time. The ions form a dense cloud at the center of the trap and both radial motions of the ions are cooled strongly by the laser. The technique can be applied to single ions and results in a tightly localized orbit. Under particular conditions the ions can be driven into a relatively large orbit with a well defined radius comparable to or larger than the laser beam diameter.

We thank M. A. van Eijkelenborg, who designed and constructed the trap, for many useful discussions. This work was supported by the EPSRC and the EU commission through the IST-QUBITS Collaboration.

[1] J. I. Cirac and P. Zoller, Phys. Rev. Lett. 74, 4091-4094 (1995).

[2] C. A. Sackett, D. Kielpinsky, B.E. King, C. Langer, V. Meyer, C. J. Myatt, M. Rowe, Q. A. Turchette, W. M. Itano, D. J. Wineland, and C. Monroe, Nature (London) 406, 256-258 (2000).

[3] C. Monroe, D. M. Meekhof, B. E. King, W. Itano, and D. J. Wineland, Phys. Rev. Lett. 75, 4714-4717 (1995).

[4] Ch. Roos, Th. Zeiger, H. Rohde, H. C. Nagerl, J. Eschner, D. Leibfried, F. Schmidt-Kaler, and R. Blatt, Phys. Rev. Lett. 83, 4713-4716 (1999).

[5] R. C. Thompson, Adv. At. Mol. Opt. Phys. 31, 63 (1993).

[6] W. M. Itano and D. J. Wineland, Phys. Rev. A 25, 35-54 (1982).

[7] R.C. Thompson and J. Papadimitriou, J. Phys. B 33, 3393-3405 (2000).

[8] L. Schweikhard and A. Marshall, J. Am. Soc. Mass Spectrom. 4, 433-452 (1993).

[9] F. Mintert and Ch. Wunderlich, Phys. Rev. Lett. 87, 257904 (2001).

[10] L. Schweikhard, M. Lindiger, and H. J. Kluge, Rev. Sci. Instrum. 61, 1055-1058 (1990).

[11] M. König, G. Bollen, H. J. Kluge, T. Otto, and J. Szerypo, Int. J. Mass Spectrom. Ion Process. 142, 95-115 (1995).

[12] Ch. Lichtenberg, G. Marx, G. Tommaseo, P. N. Ghosh, and G. Werth, Eur. Phys. J. D 2, 29-32 (1998).

[13] W. M. Itano, L. R. Brewer, D. J. Larson, and D. J. Wineland, Phys. Rev. A 38, 5698-5706 (1988).

[14] X-P. Huang, J. J. Bollinger, T. B. Mitchell, and W. M. Itano, Phys. Rev. Lett. 80, 73-76 (1998).

[15] L. Gruber, J.P. Holder, J. Steiger, B. R. Beck, H.E. De Witt, J. Glassman, J. W. McDonald, D. A. Church, and D. Schneider, Phys. Rev. Lett. 86, 636-639 (2001).

[16] S. Guan, X. Xiang, and A. G. Marshall, Int. J. Mass Spectrom. Ion Process. 124, 53-67 (1993).

[17] H.F. Powell, D. M. Segal, and R.C. Thompson (to be published).

[18] M. A. van Eijkelenborg, M.E. M. Storkey, D. M. Segal, and R. C. Thompson, Int. J. Mass Spectrom. Ion Process. 188, 155-161 (1999).

[19] K. Dholakia et al., Phys. Rev. A 47, 441-448 (1993).

[20] J. J. Bollinger et al., Phys. Rev. A 48, 525-545 (1993). 